\title{
Alterações orais em crianças prematuras e de baixo peso ao nascer: a importância da relação entre pediatras e odontopediatras
}

\author{
Oral abnormalities in preterm and low birth weight infants: the importance \\ of the relationship between pediatricians and pediatric dentists
}

Michele Baffi Diniz', Cármen Regina Coldebella², Angela Cristina C. Zuanon ${ }^{3}$, Rita de Cássia L. Cordeiro ${ }^{3}$

\section{RESUMO}

Objetivo: Apresentar uma revisão de literatura sobre as alterações orais em crianças prematuras e de baixo peso ao nascer.

Fontes de dados: Foram selecionados artigos em inglês e português, desde 1976 até 2009, pesquisados no PubMed, Lilacs e na Bibliografia Brasileira de Odontologia (BBO), além de livros e consensos nacionais e internacionais. As palavras-chave utilizadas foram: "prematuro", "recém-nascido de baixo peso", "cárie dentária”, "hipoplasia do esmalte dentário", "manifestações bucais e "dentição primária”. Selecionaram-se artigos avaliando incidência, prevalência e etiologia das alterações orais, além de revisões de literatura e relatos de casos clínicos.

Síntese dos dados: As alterações orais mais comuns em crianças prematuras e de baixo peso ao nascer são os defeitos no esmalte dentário (hipoplasias e hipocalcificações), a predisposição ao desenvolvimento de lesões de cárie dentária, as alterações na cronologia de erupção dentária e as alterações no palato com consequente aparecimento de maloclusão. Outras alterações também são relatadas, como diferenças nas dimensões das coroas dentárias e na espessura e porosidade do esmalte dentário. A interação entre pediatras e odontopediatras é fundamental no manejo dessas crianças.

Conclusões: $\mathrm{O}$ conhecimento das alterações orais em crianças pré-termo e de baixo peso ao nascer por parte dos pediatras e odontopediatras favorece a atuação multidiscipli- nar com o objetivo de educar, prevenir e atenuar as possíveis mudanças físicas e dentárias nessas crianças.

Palavras-chave: prematuro; recém-nascido de baixo peso; cárie dentária; hipoplasia do esmalte dentário; manifestações bucais; dentição primária.

\section{ABSTRACT}

Objective: To review the literature review concerning oral abnormalities in preterm and low birth-weight infants.

Data sources: Articles published in English and Portuguese on this subject were selected from 1976 to 2009, browsed on PubMed, Lilacs and Brazilian Dentistry Bibliography, besides books and national and international consensus. The search used the following key-words: "preterm infant", "low birth-weight infant", "dental caries”, "dental enamel hypoplasia", "oral manifestations" and "primary dentition". Original articles related to incidence, prevalence and etiology of the oral manifestations, literature reviews and case reports were selected.

Data synthesis: The most common oral abnormalities in preterm and low birth-weight infants are dental enamel defects, such as hypoplasias and hypocalcifications, predisposition to dental caries, alterations in the chronology of tooth eruption and palate problems with consequent development of malocclusion. Other abnormalities are also reported, such as different tooth crown dimensions and dental enamel thi-
Instituição: Faculdade de Odontologia de Araraquara da Universidade Estadual Paulista "Júlio de Mesquita Filho" (Unesp), Araraquara, SP, Brasil 'Doutora em Odontopediatria pela Faculdade de Odontologia de Araraquara da Unesp; Professora Assistente do Curso de Graduação e PósGraduação em Odontologia da Universidade Cruzeiro do Sul (Unicsul), São Paulo, SP, Brasil

${ }^{2}$ Doutora em Odontopediatria pela Faculdade de Odontologia de Araraquara da Unesp; Professora Adjunto do Curso de Odontologia da Universidade Federal do Mato Grosso do Sul (UFMS), Campo Grande, MS, Brasil

${ }^{3}$ Doutora em Odontopediatria pela Faculdade de Odontologia de Araraquara da Unesp; Professora Adjunto da Faculdade de Odontologia de Araraquara da Unesp, Araraquara, SP, Brasil.
Endereço para correspondência:

Michele Baffi Diniz

Rua Humaitá, 1680 - Centro

CEP 14801-903 - Araraquara/SP

E-mail: mibdiniz@hotmail.com

Conflito de interesse: nada a declarar

Recebido em: 29/9/2009

Aprovado em: 25/4/2011 
ckness and porosity. The interaction between pediatricians and pediatric dentists is important for the management of these infants.

Conclusions: Knowledge of oral abnormalities in preterm and low birth-weight infants by pediatricians and pediatric dentists encourages multidisciplinary action, aiming to educate, prevent and decrease the potential physical and dental changes in those infants.

Key-words: infant, premature; infant, low birth weight; dental caries; dental enamel hypoplasia; oral manifestations; dentition, primary.

\section{Introdução}

A prematuridade é definida pelo nascimento antes de completar 37 semanas de gestação(1). O baixo peso ao nascer pode ser uma consequência do nascimento prematuro ou pode estar relacionado ao pequeno tamanho para a idade gestacional, ou ambos ${ }^{(1)}$. É importante ressaltar que lactentes prematuros podem ser classificados como de baixo peso ao nascimento $(<2500 \mathrm{~g})$, muito baixo peso ao nascimento $(<1500 \mathrm{~g})$ e extremo baixo peso ao nascer $(<1000 \mathrm{~g})^{(1)}$. A etiologia relacionada ao nascimento de crianças prematuras e/ou com baixo peso é complexa e multifatorial, podendo envolver fatores genéticos, obstétricos, estado nutricional, infecções, exposição tóxica, cuidados durante o prenatal, problemas sociais, econômicos e ambientais ${ }^{(2)}$.

Segundo dados do Sistema de Informação sobre Nascidos Vivos (SINASC), houve um aumento no percentual de nascimentos prematuros no Brasil, com variação de 5\%, em 1994, a 6,6\%, em $2005^{(3)}$. De acordo com os dados do Datasus do Brasil, no ano de 2007 , a proporção de nascidos vivos prematuros e nascidos a termo com baixo peso ao nascer foi de 6,6 e de $8,2 \%$, respectivamente ${ }^{(4,5)}$. Esse fato é preocupante devido aos sérios problemas de saúde que essa crianças podem apresentar durante o curso de suas vidas, como doenças respiratórias, cardiovasculares, infecciosas, gastrintestinais e neurológicas, desordens sensoriais, disfunções metabólicas, problemas no crescimento e desenvolvimento e alterações no desenvolvimento das estruturas bucais ${ }^{(6-8)}$.

Dentre as diversas alterações e disfunções orais presentes em crianças prematuras e de baixo peso ao nascer, a literatura têm mostrado aumento na incidência de defeitos de esmalte, como hipoplasia e hipomineralização(9) , aumento na incidência de cárie dentária ${ }^{(7,10,11)}$, atraso do desenvolvimento e na erupção da dentição decídua ${ }^{(8)}$ e malformação dentária e do palato ${ }^{(8)}$.

O presente trabalho teve como objetivo analisar a literatura referente às alterações orais em crianças prematuras e de baixo peso ao nascer, enfatizando a importância da interação entre pediatras e odontopediatras, a qual pode proporcionar a essas crianças melhorias em suas condições de saúde bucal.

O levantamento dos dados contidos nesta revisão da literatura foi realizado por meio das bases de dados PubMed, Lilacs e Bibliografia Brasileira de Odontologia (BBO), além de livros e consensos nacionais e internacionais. As palavraschave utilizadas foram: "prematuro", "recém-nascido de baixo peso", "cárie dentária", "hipoplasia do esmalte dentário", "manifestações bucais" e "dentição primária”. Foram selecionados artigos de pesquisa avaliando incidência, prevalência e etiologia das alterações orais cuja metodologia e discussão estavam bem estruturadas, além de revisões de literatura e relatos de casos clínicos publicados entre 1976 e 2009 em revistas nacionais e internacionais na área de Medicina e Odontologia. A revisão de literatura deste trabalho foi redigida em tópicos, com o objetivo de facilitar o acesso e a consulta para os profissionais da saúde.

\section{Alterações orais}

\section{Defeitos no esmalte dentário}

Crianças prematuras e de baixo peso ao nascer têm apresentado alta prevalência de defeitos no esmalte dentário, como a hipoplasia e hipocalcificação, em ambas as dentições ${ }^{(12)}$, cuja incidência varia entre 66 e $96 \%{ }^{(8,13,14)}$.

A hipoplasia do esmalte é a formação insuficiente da matriz orgânica do esmalte dos dentes, resultante de lesão de suas células formadoras, os ameloblastos. De acordo com a intensidade e a extensão da agressão, podem surgir lesões discretas do ponto de vista clínico, sob a forma de um ou vários sulcos estreitos na superfície do esmalte ou lesões graves, com o aspecto de depressões profundas, dispostas horizontalmente em torno do dente ${ }^{(12,13)}$.

A hipocalcificação (ou opacidade) resulta de fatores que interferem no processo de calcificação dentária - como a imaturidade de órgãos como fígado, rins e glândulas da paratireoide - em metabolizar o cálcio. Essa alteração pode ser visualizada como uma anormalidade na translucidez do esmalte com áreas branco-opacas, amareladas ou acastanhadas, sem perda de continuidade da superfície do esmalte ${ }^{(12,13)}$.

$\mathrm{O}$ exato mecanismo e os possíveis fatores etiológicos relacionados com o desenvolvimento de defeitos no esmalte 
dentário dessas crianças ainda não são totalmente conhecidos. Sugere-se que a suplementação de cálcio e fósforo insuficiente possa aumentar o risco de defeitos no esmalte ${ }^{(9)}$.

Seow ${ }^{(15)}$ observou o envolvimento de fatores sistêmicos e locais na patogênese desses defeitos de desenvolvimento. Dentre os distúrbios sistêmicos, citam-se os nascimentos traumáticos, infecções, desordens nutricionais, doenças metabólicas e medicamentos; dentre os fatores locais, o uso de laringoscópio e intubação endotraqueal, prolongada ventilação mecânica e nutrição por sonda oral, fraturas mandibulares, trauma cirúrgico, reparo de fendas lábio-palatinas, irradiação e infecções locais.

De acordo com Caixeta e Corrêa ${ }^{(16)}$, defeitos de esmalte foram observados em $66,7 \%$ das crianças intubadas por menos de duas horas, comparadas com $74,2 \%$ intubadas entre duas e 64 horas. Segundo Blackburn ${ }^{(6)}$, a intubação orotraqueal pode traumatizar e danificar o processo alveolar palatino e, consequentemente, alterar a dentição decídua, principalmente os incisivos centrais e laterais superiores.

É importante ressaltar que a mineralização dentária decídua inicia-se no quarto mês de gestação e se completa por volta de um ano de idade. Mellander et a $l^{(17)}$ avaliaram crianças com baixo peso ao nascer com o objetivo de determinar se os distúrbios de mineralização do esmalte de dentes decíduos refletem uma nutrição neonatal inadequada. Os autores observaram que defeitos na mineralização de dentes decíduos nessas crianças parecem depender da quantidade de leite materno ingerido na primeira semana de vida, refletindo nutrição perinatal subótima de cálcio. Entretanto, a criança prematura pode apresentar sérios distúrbios no metabolismo do cálcio durante o período neonatal, devido não somente a suas características fisiológicas, mas também por complicações na gravidez, parto ou no período neonatal. De acordo com os autores, o monitoramento nutricional precoce dessas crianças favorece o desenvolvimento normal dos tecidos dentários duros. Recentemente, Lunardelli e Peres ${ }^{(18)}$ observaram que crianças prematuras e crianças que não foram amamentadas no peito apresentaram risco aumentado para o desenvolvimento de defeitos no esmalte dentário.

\section{Risco de cárie dentária}

Crianças prematuras e com baixo peso ao nascer são mais suscetíveis ao desenvolvimento de cárie, uma vez que apresentam alguns fatores de risco, como maior prevalência de defeitos no esmalte dentário, longo período de intubação, aleitamento artificial e maior exposição à sacarose, nível socioeconônico baixo, presença de bactérias na cavidade oral, hábitos incorretos de higiene oral e estado nutricional ${ }^{(19-21)}$.

A associação entre baixo peso ao nascer e lesão de cárie pode ser explicada pela debilitada resposta imune que essas crianças apresentam até os cinco anos de idade ${ }^{(22)}$. Assim, elas seriam mais suscetíveis a infecção por Streptococcus mutans durante a janela de infectividade ${ }^{(22,23)}$. Wan et al ${ }^{(23)}$ observaram que essa alta prevalência, até 4,4 vezes maior, pode ser explicada pela presença de hipoplasia de esmalte e alto consumo de açúcar ( $>3 x /$ dia) .

Entretanto, outros estudos não observaram associação entre prematuridade e alta prevalência de cárie dentária na dentição decídua ${ }^{(11,24)}$. Shulman ${ }^{(11)}$ ainda ressalta que a hipomineralização não deve ser considerada um fator que predispõe ao desenvolvimento lesões de cárie na dentição decídua.

Os resultados são controversos para a dentição permanente. Nicolau et a $l^{(10)}$ descreveram uma possível associação entre baixo peso ao nascer e lesões de cárie. Entretanto, Saraiva et $a l^{(7)}$ não encontraram indícios dessa associação.

\section{Alterações na cronologia de erupção dentária}

A prematuridade e o baixo peso ao nascer têm sido descritos como possíveis causas para o atraso na erupção dentária ${ }^{(25-27)}$. Entretanto, ainda não se sabe como a erupção dentária é afetada por essa condição.

Seow et al ${ }^{(25)}$ verificaram que crianças com muito baixo peso ao nascer apresentaram poucos dentes decíduos erupcionados entre seis e 11 meses de idade e entre 12 e 17 meses, comparadas a crianças com baixo peso ao nascer ou a crianças com peso normal ao nascer.

Drummond et al ${ }^{(26)}$ observaram que, aos 30 meses de idade, $50 \%$ das crianças prematuras apresentaram erupção completa dos dentes decíduos, enquanto esse número foi de $67 \%$ nas nascidas a termo. Viscardi $e t a^{(27)}$ verificaram que crianças prematuras têm, em média, seus dentes decíduos erupcionados na $39^{\mathrm{a}}$ semana de vida pós-natal, enquanto que as que nascem a termo, na $30^{\mathrm{a}}$ semana. Os autores ainda relatam que o período de permanência da cânula traqueal, durante a internação neonatal, é o maior responsável pelo atraso na erupção dos dentes decíduos. Entretanto, outros fatores podem resultar em erupção tardia, como distúrbios endócrinos, hipotireoidismo e disostose cleidocranial, ou em erupção avançada, como a puberdade precoce ${ }^{(25)}$.

Harila-Kaera $e t$ al ${ }^{(28)}$ sugerem que a erupção precoce em crianças nascidas prematuramente pode ser observada em dentes que sofreram, no período neonatal, influência de 
doenças sistêmicas, durante as fases de pré-mineralização e pré-erupção, como os incisivos e primeiros molares permanentes.

Em um estudo realizado em 2005, Caixeta e Corrêa ${ }^{(16)}$ analisaram crianças prematuras entre seis meses a seis anos de idade. Embora tenham observado que os dentes decíduos irromperam em tempo normal, o número de dentes presentes até os 36 meses foi menor do que os encontrados em crianças nascidas a termo.

Recentemente, Ramos et al ${ }^{(29)}$ avaliaram a erupção dos dentes decíduos em crianças prematuras e a termo de peso normal, baixo peso ou muito baixo peso ao nascimento. Os autores observaram erupção dentária tardia em crianças prematuras e de muito baixo peso ao nascer em relação à idade cronológica. Dessa forma, concluiu-se que o atraso na erupção pode ser simplesmente devido ao nascimento precoce e não ao desenvolvimento dentário tardio.

\section{Alterações no palato e arcadas dentárias}

Estudos têm mostrado que algumas alterações nas estruturas orais, como a formação de palato arqueado, fissurado ou ogival na região de palato duro e as alterações no desenvolvimento das arcadas dentárias, são mais frequentes em crianças prematuras ${ }^{(30,31)}$. A pressão exercida pela cânula orotraqueal ou pelo laringoscópio seria responsável por tais alterações, inibindo o crescimento normal dessas estruturas. A literatura sugere o emprego de posicionadores intraorais adequados para estabilização da cânula orotraqueal, reduzindo o trauma às vias respiratórias superiores e prevenindo as deformidades palatinas ${ }^{(31)}$.

Alguns trabalhos sugerem que o tempo de intubação influencia na gravidade das alterações orais. Duke et al ${ }^{(30)}$ observaram palato profundo em neonatos após 70 dias de intubação e uma fissura correspondente à forma do tubo orotraqueal na linha média do palato duro após 90 dias. Erenberg e Nowak ${ }^{(31)}$ notaram que as anormalidades palatais começaram a se desenvolver após 12 horas de intubação orotraqueal. Entretanto, Macey-Dare et al ${ }^{(32)}$ não evidenciaram associação entre o tempo de intubação e a alteração na morfologia do palato, como estrias, assimetrias e palato profundo, mas observaram correlação positiva entre a alteração e a intubação oral.

Crianças de extremo baixo peso ao nascer apresentam maior incidência de fendas palatais, comparadas às que nasceram com mais de $1 \mathrm{~kg}^{(33)}$. Dois fatores influenciam negativamente a gravidade das fissuras palatais: o tempo em que a criança permanece intubada e o movimento de sucção, pois a língua exerce um papel importante no desenvolvimento anatômico do palato. De acordo com Duke et $a^{(30)}$, a remoção da cânula e a movimentação normal da língua possibilitam uma recuperação da normalidade anatômica do palato em até dois anos, dependendo do dano causado anteriormente.

Sabe-se que alterações na morfologia do palato podem resultar em aumento na prevalência de maloclusões, como a mordida cruzada, necessitando de intervenção ortodôntica ${ }^{(34)}$. Entretanto, Fadavi et al ${ }^{(35)}$ não observaram diferenças significantes na prevalência de mordida cruzada na dentição decídua entre crianças prematuras e nascidas a termo. Almeida et $a^{(36)}$ não notaram diferenças significativas no padrão morfológico dos roletes gengivais de recém-nascidos prematuros, comparados aos nascidos a termo, sugerindo que a prematuridade não interfere no tipo de relação oclusal no período pré-dentário.

Harila-Kaera et a ${ }^{(37)}$ observaram que crianças prematuras podem apresentar maior predisposição aos fatores etiológicos que facilitam o desenvolvimento de mordida aberta anterior, como a adaptação precoce às funções orais extrauterinas (hábitos de sucção e nutrição), infecções respiratórias, respiração oral ou respiração nasal inadequada e hábitos orais não nutritivos. Os autores enfatizam a importância de estimular precocemente a atividade funcional dos músculos mastigatórios.

Recentemente, uma revisão sistemática observou alteração na morfologia palatal em crianças nascidas prematuramente, sendo a intubação oral o fator contribuinte para tal alteração. Entretanto, os autores não encontraram evidências para relacionar a presença de maloclusões com a prematuridade ${ }^{(34)}$.

\section{Outras alterações nos tecidos dentários duros}

Outra alteração que também é citada na literatura diz respeito à espessura do esmalte dentário nos dentes decíduos de crianças prematuras. Um estudo recente observou microscopicamente que o esmalte dentário de incisivos centrais superiores decíduos dessas crianças é irregular e significativamente mais fino, comparado ao esmalte de crianças nascidas a termo ${ }^{(38)}$. Esse fato pode ser explicado principalmente pelo crescimento prenatal reduzido, que resulta em dimensões menores dos dentes decíduos.

Crianças prematuras também podem apresentar alterações nas dimensões das coroas dentárias ${ }^{(39-41)}$. Seow e $\operatorname{Wan}^{(41)}$ observaram que crianças prematuras com baixo e muito baixo peso ao nascer tinham dentes decíduos com dimensões intermediárias e menores, respectivamente, quando comparadas 
às crianças com peso normal. Entretanto, Paulsson et al ${ }^{(34)}$, em uma revisão sistemática, não encontraram evidências de tais alterações. Harila et al ${ }^{(40)}$ notaram que o período mais curto de gestação não se associa ao tamanho reduzido da coroa dentária na dentição decídua, como sugerido por outros estudos.

Com relação aos dentes permanentes, sugere-se que fatores ambientais determinem as dimensões das coroas dentárias ${ }^{(39)}$. Crianças nascidas prematuramente apresentam coroas com dimensões reduzidas. Entretanto, um aumento na dimensão das coroas foi observado em garotos da raça caucasiana e garotas da raça negra.

Além disso, outras alterações em crianças prematuras com baixa idade gestacional têm sido descritas na literatura, como aumento na porosidade do esmalte, principalmente de molares e caninos decíduos; presença de linhas incrementais no esmalte pós-natal devido a distúrbios no metabolismo de cálcio $^{(42)}$ e dilaceração da coroa dentária por forças traumáticas locais da laringoscopia e intubação orotraqueal ${ }^{(43)}$.

\section{Hábitos orais nutritivos e não nutritivos}

Sabe-se que o leite materno, além de reduzir a morbidade em crianças prematuras, apresenta propriedades nutritivas e imunológicas, estimula a maturação gastrintestinal do bebê, melhora o desenvolvimento cognitivo e psicomotor, aumenta o vínculo afetivo mãe/filho e estimula o desenvolvimento orofacial $^{(44)}$. Os movimentos adequados da musculatura oral previnem alterações de hipodesenvolvimento, maloclusões e problemas articulatórios. Além disso, previnem a instalação de hábitos de sucção não fisiológica, que poderão influenciar negativamente o desenvolvimento normal da função e oclusão das crianças $^{(45)}$.

Hernandez $^{(46)}$ ressalta algumas características nos recémnascidos pré-termos que geram dificuldades na função de sucção e alimentação e, consequentemente, na implementação do aleitamento materno, como hipotonia em extensão, excessiva abertura de boca, ausência de almofadas de gordura nas bochechas, dificuldade quanto à estabilidade da mandíbula, dificuldades respiratórias, vedamento labial insuficiente e reflexos orais incompletos ou ausentes.

Sabe-se que o aleitamento artificial é empregado por meio de mamadeiras com os substitutos do leite materno, muitos dos quais incluem açúcares em suas formulações. Dessa forma, o aleitamento artificial por longos períodos, principalmente durante o período noturno, aumenta o risco de desenvolvimento da lesão de cárie dentária e da instalação de hábitos deletérios, com consequente instalação de maloclusões ${ }^{(44)}$. Em vista disso, os autores enfatizam a importância da amamentação natural aos recém-nascidos prematuros e afirmam que, mesmo sendo um desafio, é possível realizá-la desde que haja determinação e suporte da família, da sociedade e, em especial, dos profissionais de saúde. O aleitamento materno deve ser encorajado, sem descartar a necessidade do uso de aditivos ao leite materno, que possibilitam ao recém-nascido de muito baixo peso ao nascer crescer de modo adequado, pois, apesar de o leite da mães de recém-nascidos prematuros apresentar maiores concentrações de nitrogênio, lípidios, ácidos graxos, cálcio, fósforo e sódio, ao final do primeiro mês de lactação estas tornam-se semelhantes à concentração desses componentes no leite de mães de recém-nascidos a termo, limitando a oferta energética e proteica ${ }^{(47)}$.

\section{Interação entre pediatras e odontopediatras}

O peso ao nascer e/ou a idade gestacional ao nascer são fatores que influenciam o estado de saúde e as chances de sobrevivência das crianças ${ }^{(1)}$. Nos últimos anos, houve um avanço nos cuidados neonatais, o que permite que prematuros de baixo ou muito baixo peso contem com maiores taxas de sobrevida ${ }^{(14)}$. Contudo, a condição bucal dessas crianças tem recebido pouca atenção por parte dos familiares e da equipe de saúde, uma vez que o desenvolvimento físico e psicológico é visto como prioritário ${ }^{(14)}$.

Considerando que a educação em saúde bucal deveria iniciar-se logo após o nascimento da criança, torna-se importante uma abordagem integrada interdisciplinar entre pediatras e odontopediatras, com integração entre saúde bucal e geral $^{(48)}$. Uma vez que os pediatras são os primeiros da equipe de saúde a terem contato com os bebês, eles devem atuar na conscientização das famílias quanto aos possíveis problemas bucais, principalmente a cárie dentária.

Freire et al ${ }^{(49)}$ demonstram que os médicos, em sua maioria, procedem de forma adequada quanto às orientações sobre higiene oral; entretanto, desconhecem em parte a etiologia da cárie. Pierce et al ${ }^{(50)}$ verificaram que o exame dentário pode ser facilmente integrado na prática pediátrica. Assim, sugere-se que os pediatras são aptos a orientar os pais sobre os cuidados preventivos relacionados à cárie dentária, incluindo hábitos dietéticos, higiene oral e uso do flúor.

Tendo em vista que crianças nascidas prematuramente e de baixo peso apresentam defeitos na estrutura do esmalte dentário, Caixeta e Corrêa ${ }^{(16)}$ aconselham exames pós-natais periódicos e orientações por parte dos pediatras para melhorar 
a absorção de cálcio em prematuros, evitando-se, assim, comprometimentos futuros na fase de deposição e mineralização do esmalte dentário.

Sabe-se que crianças prematuras necessitam de atenção odontológica especial, pois são mais vulneráveis a situações estressantes $^{(24)}$. Embora essa atitude nem sempre ocorra, o ideal seria que os pediatras iniciassem a atenção à saúde bucal dessas crianças e, então, encaminhassem as mesmas para um tratamento odontológico especializado.

\section{Conclusões}

A presente revisão de literatura mostrou que, dentre as alterações orais, os defeitos no esmalte dentário, a predisposição ao desenvolvimento de lesões de cárie dentária e a alteração na cronologia de erupção dentária são condições que merecem ênfase em crianças prematuras e de baixo peso ao nascer. Além disso, é preciso prestar atenção na associação entre intubação orotraqueal nessas crianças e as possíveis

\section{Referências bibliográficas}

1. Edmond K, Bahl R. Optimal feeding of low-birth-weight infants: technical review. Geneva: World Health Organization; 2006.

2. Williams CE, Davenport ES, Sterne JA, Sivapathasundaram V, Fearne JM, Curtis MA. Mechanisms of risk in preterm low-birthweight infants. Periodontol 2000;23:142-50.

3. Silveira MF, Santos IS, Matijasevich A, Malta DC, Duarte EC. Nascimentos pré-termo no Brasil entre 1994 e 2005 conforme o Sistema de Informações sobre Nascidos Vivos (SINASC). Cad Saude Publica 2009;25:1267-75.

4. Brasil - Ministério da Saúde. DATASUS [homepage on the Internet]. Informações de Saúde - Estatísticas Vitais - Mortalidade e Nascidos Vivos: nascidos vivos desde 1994 [cited 2010 Apr 15]. Available from: http://tabnet. datasus.gov.br/cgi/deftohtm.exe?sinasc/cnv/nvuf.def

5. Monteiro CA, Benicio MH, Ortiz LP. Secular trends in birth weight in S. Paulo city, Brazil (1976-1998). Rev Saude Publica 2000;34:26-40.

6. Blackburn S. Problems of preterm infants after discharge. J Obstet Gynecol Neonatal Nurs 1995;24:43-9.

7. Saraiva MC, Chiga S, Bettiol H, Silva AA, Barbieri MA. Is low birthweight associated with dental caries in permanent dentition? Paediatr Perinat Epedemiol 2007;21:49-56.

8. Seow WK. Effects of preterm birth on oral growth and development. Aust Dent J 1997;42:85-91.

9. Seow WK, Brown JP, Tudehope DI, O'Callaghan M. Developmental defects in the primary dentition of low birth-weight infants: adverse effects of laryngoscopy and prolonged endotracheal intubation. Pediatr Dent 1984;6:28-31.

10. Nicolau B, Marcenes W, Bartley M, Sheiham A. A life course approach to assessing causes of dental caries experience: the relationship between biological, behavioural, socio-economic and psychological conditions and caries in adolescents. Caries Res 2003;37:319-26.

11. Shulman JD. Is there an association between low birth weight and caries in the primary dentition? Caries Res 2005;39:161-7.

12. Pimlott JF, Howley TP, Nikiforuk G, Fitzhardinge PM. Enamel defects in prematurely born, low birth-weight infants. Pediatr Dent 1985;7:218-23. alterações no palato, podendo favorecer o desenvolvimento de maloclusões.

Os avanços nos cuidados neonatais não contribuíram para diminuir a prevalência de defeitos de esmalte e, consequentemente, de lesões de cárie nessas crianças. De acordo com Machado e Ribeiro ${ }^{(14)}$ e Barbosa et al ${ }^{(51)}$, o aumento na prevalência dos defeitos de esmalte descritos nos últimos anos é decorrente dos altos índices de sobrevida nessas crianças, favorecendo a realização de pesquisas abordando tal relação. Espera-se que o entendimento das alterações orais nessas crianças, tanto por parte dos pediatras como dos odontopediatras, favoreça a atuação interdisciplinar, proporcionando o adequado manejo dessas crianças e a orientação apropriada aos pais. É importante ressaltar que programas de atenção integral à saúde da criança também devem ser desenvolvidos para melhorar os cuidados pré-natal, neonatal e pós-natal, incluindo programas de incentivo à amamentação natural com o objetivo de educar, prevenir e atenuar as possíveis mudanças físicas e dentárias nessas crianças.

13. Aine L, Backström MC, Mäki R, Kuusela AL, Koivisto AM, Ikonen RS et al. Enamel defects in primary and permanent teeth of children born prematurely. J Oral Pathol Med 2000;29:403-9.

14. Machado FC, Ribeiro RA. Enamel defects and dental caries in premature and/ or low birthweight children. Pesq Bras Odontoped Clin Integr 2004;4:243-7.

15. Seow WK. Enamel hypoplasia in the primary dentition: a review. ASDC J Dent Child 1991;58:441-52.

16. Caixeta FF, Corrêa MS. Os defeitos do esmalte e a erupção dentária em crianças prematuras. Rev Assoc Med Bras 2005;51:195-9.

17. Mellander M, Norén JG, Fredén H, Kjellmer I. Mineralization defects in deciduous teeth of low birthweight infants. Acta Paediatr Scand 1982;71:727-33.

18. Lunardelli SE, Peres MA. Breast-feeding and other mother-child factors associated with developmental enamel defects in the primary teeth of Brazilian children. J Dent Child 2006;73:70-8.

19. Fadavi S, Punwani I, Vidyasagar D. Prevalence of dental caries in prematurelyborn children. J Clin Pediatr Dent 1993;17:163-5.

20. Burt BA, Pai S. Does low birthweight increase the risk of caries? A systematic review. J Dent Educ 2001;65:1024-7.

21. Saraiva MC, Bettiol H, Barbieri MA, Silva AA. Are intrauterine growth restriction and preterm birth associated with dental caries? Community Dent Oral Epidemiol 2007;35:364-76.

22. Chandra RK. Nutrition and the immune system from birth to old age. Eur $\mathrm{J}$ Clin Nutr 2002;56:S73-6.

23. Wan AK, Seow WK, Purdle DM, Bird PS, Walsh LJ, Tudehope DI. Immunoglobulins in saliva of preterm and full-term infants. A longitudinal study from 0-18 months of age. Oral Microbiol Immunol 2003;18:72-8.

24. Brogårdh-Roth $S$, Stjernqvist $\mathrm{K}$, Matsson L. Dental behavioural management problems and dental caries prevalence in 3- to 6-year-old Swedish children born preterm. Int J Paediatr Dent 2008;18:341-7.

25. Seow WK, Humphrys C, Mahanonda R, Tudehope DI. Dental eruption in low birth-weight prematurely born children: a controlled study. Pediatr Dent 1988;10:39-42. 
26. Drummond BK, Ryan S, O'Sullivan EA, Congdon P, Curzon ME. Enamel defects of the primary dentition and osteopenia of prematurity. Pediatr Dent 1992;14:119-21.

27. Viscardi RM, Romberg E, Abrams RG. Delayed primary tooth eruption in premature infants: relationship to neonatal factors. Pediatr Dent 1994;16:23-8.

28. Harila-Kaera V, Heikkinen T, Alvesalo L. The eruption of permanent incisors and first molars in prematurely born children. Eur J Orthod 2003;25:293-9.

29. Ramos SR, Gugisch RC, Fraiz FC. The influence of gestational age and birth weight of the newborn on tooth eruption. J Appl Oral Sci 2006;14:228-32.

30. Duke PM, Coulson JD, Santos JI, Johnson JD. Cleft palate associated with prolonged orotracheal intubation in infancy. J Pediatr 1976;89:990-1.

31. Erenberg A, Nowak AJ. Palatal groove formation in neonates and infants with orotracheal tubes. Am J Dis Child 1984;138:974-5.

32. Macey-Dare LV, Moles DR, Evans RD, Nixon F. Long-term effect of neonatal endotracheal intubation on palatal form and symmetry in 8-11-year-old children. Eur J Orthod 1999;21:703-10.

33. Molteni RA, Bumstead DH. Development and severity of palatal grooves in orally intubated newborns. Effect of 'soft' endotracheal tubes. Am J Dis Child 1986;140:357-9.

34. Paulsson L, Bondemark L, Söderfeldt B. A systematic review of the consequences of premature birth on palatal morphology, dental occlusion, tooth-crown dimensions, and tooth maturity and eruption. Angle Orthod 2004;74:269-79.

35. Fadavi S, Adeni S, Dziedzic K, Punwani I, Vidyasagar D. The oral effects of orotracheal intubation in prematurely born preschoolers. ASDC J Dent Child 1992;59:420-4

36. Almeida MN, Siqueira TO, Ribeiro SM. Evaluation of the occlusion in pre-dental period in premature born in Santa Casa de Misericórdia do Pará. Rev Para Med 2007;21:31-6.

37. Harila-Kaera V, Grön M, Heikkinen T, Alvesalo L. Sagittal occlusal relationships and asymmetry in prematurely born children. Eur J Orthod 2002;24:615-25.

38. Seow WK, Young WG, Tsang AK, Daley T. A study of primary dental enamel from preterm and full-term children using light and scanning electron microscopy. Pediatr Dent 2005;27:374-9.

39. Harila-Kaera V, Heikkinen T, Alvesalo L, Osborne RH. Permanent tooth crown dimensions in prematurely born children. Early Hum Dev 2001;62:131-47.

40. Harila V, Heikkinen T, Alvesalo L. Deciduous tooth crown size in prematurely born children. Early Hum Dev 2003;75:9-20.

41. Seow WK, Wan A. A controlled study of the morphometric changes in the primary dentition of pre-term, very-low-birthweight children. J Dent Res 2000;79:63-9.

42. Rythén M, Norén JG, Sabel N, Steiniger F, Niklasson A, Hellström A et al. Morphological aspects of dental hard tissues in primary teeth from preterm infants. Int J Paediatr Dent 2008;18:397-406.

43. Seow WK, Perham S, Young WG, Daley T. Dilaceration of a primary maxillary incisor associated with neonatal laryngoscopy. Pediatr Dent 1990;12:321-4.

44. Braga DF, Machado MM, Bosi ML. Achieving exclusive breastfeeding of premature babies: the perceptions and experience of women from public health services. Rev Nutr 2008;21:293-302.

45. Van Der Laan T. A importância da amamentação no desenvolvimento facial infantil. Pró-fono 1995;7:3-5.

46. Hernandez AM. Atuação fonoaudiológica em neonatologia: uma proposta de intervenção. In: Andrade CR. Fonoaudiologia em berçário normal e de risco. São Paulo: Lovise; 1996. p. 43-98.

47. Mataloun MM, Leone CR, Ono N, Vaz FA. Repercussões neonatais do uso de leite materno com aditivos e fórmula para pré-termo em recém-nascidos de muito baixo peso ao nascer. Pediatria (São Paulo) 2004;26:247-56.

48. Sheiham $A$. The role of the dental team in promoting dental health and general health through oral health. Int Dent J 1992;42:223-8.

49. Freire MC, Macedo RA, Silva WH. Knowledge, attitudes and practice of pediatricians in relation to oral health. Pesqui Odontol Bras 2000;14:39-45.

50. Pierce KM, Rozier RG, Vann Jr WF. Accuracy of pediatric primary care providers' screening and referral for early childhood caries. Pediatrics 2002;109:e82.

51. Barbosa DM, Lemos LV, Banzi EC, Myaki SI. Prevalência de hipoplasia do esmalte em dentes decíduos de crianças nascidas prematuras. Rev Odontol Unesp 2008;37:261-5 